\title{
Erratum: Pulse reverse engineering for controlling two-level quantum systems [Phys. Rev. A 101, 023822 (2020)]
}

\author{
Du Ran®, Wu-Jiang Shan, Zhi-Cheng Shi, Zhen-Biao Yang, Jie Song, and Yan Xia
}

(Received 8 June 2020; published 17 July 2020)

DOI: 10.1103/PhysRevA.102.019902

It has been brought to our attention that, although we have pointed out the differences between our original paper and Ref. [1], we find that the discussions on the differences are not very clear. In this Erratum, we want to provide more details about the differences and highlight the earlier results in several respects.

First, in Sec. III of our original paper, for facilitating comparison with the existing techniques, we consider a similar predetermined dynamics with Refs. [1,2] to show that the scheme works well for both closed and open quantum systems. To be specific, we use the population control function $w(t)$ and the coherence control function $C_{A}(t)$ to show the system dynamics, whose notations are equivalent to the ground-state population related function $f(t)$ and coherence related function $h(t)$ in Refs. [1,2]. It is worth mentioning that, although the function $w(t)$ is the same with $f(t)$, the coherence control function $C_{A}(t)$ in our original paper is different from $h(t)$ in Ref. [1] because the inner components in $C_{A}(t)$ include $u(t)$ and $v(t)$ and we can choose different parameters in $v(t)$ to control $C_{A}(t)$. As a consequence, different $v(t)$ 's will result in a different trajectory on the Bloch sphere, whereas Ref. [1] may be invalid when the phase of coherence is time varying, i.e., not all the trajectories are achievable.

Second, for the simulation examples in Sec. III B of our original paper, we first consider the influence of pure dephasing, then consider both dephasing and thermal noises. Similar investigations have also been involved in Secs. III A and III B of Ref. [1], respectively. In order to show the advantages of the scheme in our original paper, we choose the same parameters as Ref. [1] to carry out numerical simulations, which are shown in Fig. 9 of our original paper. Note that, although the population related function $w(t)$ in our original paper is the same as the function $f(t)$ in Refs. [1,2], the same value of $a_{i}\left(a_{f}\right)$ will result in a different initial state (final state). For example, $a_{i}=0.2$ results in the population of ground-state $P_{g}=0.4$ in our original paper whereas it leads to the population of $P_{g}=0.2$ in Ref. [1]. Furthermore, the evolution of trajectories is quite different from Ref. [1] because we have a specific function of $v(t)$. Thus, all simulation results in our original paper are different from that in Ref. [1], even though we use the same parameters.

Third, in our original paper, it is possible to predict the population and coherence at each moment exactly for both closed and open quantum systems, and the simulation results indicate the existence of steady states, which is similar to the case shown in Sec. III C of Ref. [1]. The results obtained in Ref. [1] show that there are unachievable final populations (see Fig. 7 of Ref. [1]) and the region of accessible populations changes with an increment of $\Gamma$. For the scheme in our original paper, the accessible region of final states also varies with $\Gamma$, but the accessible region of the final states in Fig. 9(a) is much larger than that in Ref. [1] due to the different control strategies and different evolution of trajectories. We think that these aspects should be better referenced and highlighted.

Finally, we want to stress that the pulse design method in our original paper is different from the previous one [1] since the pulse $E(t)$ is designed based on reversely solving the optical Bloch equation so that the scheme in our original paper can be easily applied to the system model involving time-varying transition frequency [3-5], which might be difficult by the method in Refs. [1,2].

These additional discussions do not affect the results and conclusions of the original paper.

[1] I. Medina and F. L. Semião, Phys. Rev. A 100, 012103 (2019).

[2] N. V. Golubev and A. I. Kuleff, Phys. Rev. A 90, 035401 (2014).

[3] S. Ibáñez, A. P. Conde, D. Guéry-Odelin, and J. G. Muga, Phys. Rev. A 84, 013428 (2011).

[4] S. Ibáñez, Y. C. Li, X. Chen, and J. G. Muga, Phys. Rev. A 92, 062136 (2015).

[5] D. Ran, B. Zhang, Y.-H. Chen, Z.-C. Shi, Y. Xia, R. Ianconescu, J. Scheuer, and A. Gover, Opt. Lett. 45, 3597 (2020). 